\title{
ON EMBEDDING POLYHEDRA AND MANIFOLDS
}

\author{
BY \\ KREŠO HORVATIĆ
}

\begin{abstract}
It is well known that every $n$-polyhedron PL embeds in a Euclidean $(2 n+1)$-space, and that for PL manifolds the result can be improved upon by one dimension. In the paper are given some sufficient conditions under which the dimension of the ambient space can be decreased. The main theorem asserts that, for there to exist an embedding of the $n$-polyhedron $X$ into $2 n$-space, it suffices that the integral cohomology group $H^{n}(X-\operatorname{Int} A)=0$ for some $n$-simplex $A$ of a triangulation of $X$. A number of interesting corollaries follow from this theorem. Along the line of manifolds the known embedding results for PL manifolds are extended over a larger class containing various kinds of generalized manifolds, such as triangulated manifolds, polyhedral homology manifolds, pseudomanifolds and manifolds with singular boundary. Finally, a notion of strong embeddability is introduced which allows us to prove that some class of $n$-manifolds can be embedded into a $(2 n-1)$-dimensional ambient space.
\end{abstract}

1. Introduction. It was known early [14] that every $n$-dimensional polyhedron can be piecewise linearly embedded into a Euclidean space of dimension $2 n+1$, and that for piecewise linear manifolds this result can be improved upon by one dimension. On the other hand, there are counterexamples showing that these results are, in general, the best possible ([5], [6], [19]). So the natural problem arises as to characterizing those polyhedra and manifolds for which the dimension of the ambient space can be decreased.

In this paper we present a number of sufficient conditions under which this is a case.

The paper is a part of the author's doctoral dissertation, written during his stay at the Department of Mathematics, University of Georgia, Athens, U.S.A., under the direction of Professor C. H. Edwards, Jr.

2. Preliminaries. We work entirely within the piecewise linear (abbreviation: PL) category, i.e. all objects considered are assumed to have PL structures, and all the maps will be PL. The terminology and notation throughout this paper will be the same as in Zeeman's notes [24], with the substitution of today's more common PL for his prefix "poly". Also, we assume familiarity with the definitions and results of [24].

The only ambient space in our program will be the Euclidean space $E^{n}$ of dimension $n$, topologized by the standard metric and carrying the standard PL structure. For our purposes, a complex will be always a finite simplicial complex, and hence

Received by the editors August 6, 1969.

AMS 1970 subject classifications. Primary 57C35.

Key words and phrases. PL category, PL embeddings, polyhedra, PL manifolds, generalizations of manifolds, strong embeddability. 
contained in some Euclidean space. Consequently, a polyhedron will be an underlying space of such a complex, and therefore compact. The notation $n$-polyhedron means that the polyhedron is of dimension $n$.

We recall that a PL $n$-ball $B^{n}\left(n\right.$-sphere $\left.S^{n}\right)$ is a polyhedron PL homeomorphic to the $n$-simplex (boundary of $(n+1)$-simplex). Then, a PL $n$-manifold is a polyhedron $M$ in which every point has a closed neighborhood which is a PL $n$-ball. The boundary $\mathrm{Bd} M$ (or $\partial M$ ) and the interior Int $M$ of the PL manifold $M$ are defined to be the same as those of the underlying topological manifold. Alternatively, to give PL definitions for these notions, we first observe that such a manifold $M$ always has a triangulation $K$ with respect to which the underlying space of the link of every vertex is either a PL $(n-1)$-sphere or a PL $(n-1)$-ball. Then, the point $x \in M$ belongs to the boundary of $M$ if there is a subdivision of $K$ in which $x$ is a vertex having a ball as a link. Otherwise, $x$ belongs to the interior of $M$. Of course, it can be shown that this definition is invariant, i.e. it does not depend on the particular triangulation or subdivision. If $\mathrm{Bd} M \neq 0$, we say that $M$ is a PL manifold with (nonempty) boundary. If $\mathrm{Bd} M=0, M$ is called a closed PL manifold. We will continue to use the same terminology for all other categories of manifolds defined below. Finally, let us mention that the boundary of a PL $n$-manifold with boundary is always a closed PL $(n-1)$-manifold.

A triangulated $n$-manifold is any $n$-polyhedron $X$, which is a topological manifold. The boundary and the interior of $X$ are defined in the obvious way. It is known that any such manifold is actually a PL manifold if $n \leqq 3$, and the equivalence depends on the classical Poincaré conjecture if $n=4$. There are no known examples of triangulated manifolds which are not PL.

A polyhedral homology n-manifold is a polyhedron $X$ such that the link of every vertex of a triangulation of $X$ is either acyclic or is a homology $(n-1)$-sphere, i.e., a polyhedron having the homology groups of the $(n-1)$-sphere. Furthermore, it can be proven $[16$, p. 239] that every link is a polyhedral homology $(n-1)$ manifold (notice that under our definition a homology sphere is not automatically a homology manifold). The boundary of such a manifold is then defined to be the collection of all those points, each of which has in a proper triangulation (i.e. in a triangulation in which it is a vertex) an acyclic link. The boundary of a polyhedral homology $n$-manifold is a closed polyhedral homology $(n-1)$-manifold. The use of local homology groups shows easily that every triangulated manifold is a polyhedral homology manifold, having a boundary in the new sense, which obviously agrees with the old one. There are counterexamples which show that the converse is not true in general. For example, a suspension of the "Poincaré sphere" is a polyhedral homology 4-manifold, which is not a PL one. We will need the following statement, which is easy to prove:

2.1. Proposition. Let $X$ be a closed polyhedral homology n-manifold and $A$ any $n$-simplex in the triangulation of $X$. Then, $X-\operatorname{Int} A$ is a polyhedral homology $n$ manifold with boundary $\partial A$. 
An $n$-dimensional pseudomanifold is a polyhedron $X$ such that any triangulation $K$ of $X$ satisfies the following conditions:

(1) every principal simplex of $K$ is $n$-dimensional,

(2) every $(n-1)$-simplex of $K$ is a face of at most two $n$-simplexes, and

(3) given any two $n$-simplexes $A$ and $B$ of $K$, there is a finite sequence $A=A_{0}$, $A_{1}, \ldots, A_{k}=B$ of $n$-simplexes in $K$ such that $A_{i} \cap A_{i-1}$ is a common $(n-1)$-face of $A_{i}$ and $A_{i-1}, i=1, \ldots, k$.

This definition does not depend on the particular triangulation, i.e. if the conditions above are satisfied for one triangulation of $X$, they are satisfied for any other triangulation of $X$. The boundary of a pseudomanifold is defined to be the subpolyhedron of $X$ determined by all those $(n-1)$-simplexes of $K$, each of which is a face of exactly one $n$-simplex of $K$. In general, the boundary of a pseudomanifold is not a pseudomanifold by itself. It is easy to see that every connected PL manifold, and more generally every polyhedral homology manifold, possesses properties (1)-(3) [16, p. 238]. Therefore every connected polyhedral homology manifold $X$ is a pseudomanifold, and moreover, the boundary of $X$ as a pseudomanifold is the same as the boundary of $X$ as a homology manifold [17, p. 278]. However, the converse is not true. For example, a "pinched" torus is obviously a pseudomanifold, but not a homology manifold.

Finally, an n-manifold with singular boundary is a polyhedron $X$ for which there is a PL $n$-manifold $M$ with nonempty boundary and a PL map $f: M \rightarrow X$ onto $X$ such that the singular set $S(f)$ (see [24, Chapter 6]) is contained in the boundary of $M$. The boundary of such a manifold is then defined as the subpolyhedron of $X$ which is the image under $f$ of the part of $\partial M$ on which the restriction $f / \partial M$ is $1-1$, i.e.,

$$
\partial X=\mathrm{Cl} f(\partial M-S(f))
$$

It is easy to prove that every pseudomanifold is an image of a PL ball under a map having its singular set in the boundary of the ball [4]. Therefore every pseudomanifold $X$ is a manifold with singular boundary, and further, the boundary of $X$ in the new sense and the old sense agree. The converse is not true. For example, if we identify three parts of the boundary of a disc, we will obtain a manifold with singular boundary, which is not a pseudomanifold.

Now if PLM denotes the set of all connected PL manifolds, TM the set of connected triangulated manifolds, PHM the set of connected polyhedral homology manifolds, PSM the set of pseudomanifolds, and finally SBM the set of all manifolds with singular boundary, we have the following sequence of inclusions

$$
\mathrm{PLM} \subset \mathrm{TM} \subset \mathrm{PHM} \subset \mathrm{PSM} \subset \mathrm{SBM}
$$

each of the inclusions being proper, except perhaps the first one, depending upon the truth of the conjecture that every triangulated manifold is PL. 
Therefore, any conclusion for manifolds with singular boundary will remain true for all other categories of manifolds listed above. In particular, we will need the following:

2.2. LEMMA. Every proper subpolyhedron $Y$ of the n-manifold with singular boundary $X$ collapses to some $(n-1)$-dimensional subpolyhedron of $X$.

Proof. For the notion of collapsibility see, for example, [24, Chapter 3].

By definition there is a PL manifold $M$ and PL map $f: M \rightarrow X$ onto $X$ such that $S(f) \subset \partial M$. Let $J$ be a triangulation of $M$ and $(K, L)$ a triangulation of the pair $(X, Y)$ with respect to which $f$ is simplicial.

First of all let us notice that, if $A \in K$ is any $n$-simplex, then $f^{-1}(A)$ is an $n$ simplex of $J$. For, $f^{-1}(A)$ cannot be an $(n+1)$-simplex, because $M$ is $n$-dimensional, and further $f^{-1}(A)$ cannot consist of two or more $n$-simplexes, because in this case the singular set $S(f)$ would be $n$-dimensional.

We can suppose that $L$ is $n$-dimensional; otherwise the assertion is trivial. Because $L$ is a proper subcomplex of $K$, we have $K-L \neq 0$ and then

$$
f^{-1}(K-L)=J-f^{-1}(L) \neq 0
$$

where $f^{-1}(L)$ is an $n$-dimensional subcomplex of $J$. Let $A$ be any $n$-simplex of $f^{-1}(L)$. Further, if $S \in J-f^{-1}(L)$ is any simplex, there is a principal simplex $B$, such that $S$ is a face of $B$ and $B$ is $n$-dimensional, because $M$ is a PL manifold. Clearly $B \in J-f^{-1}(L)$, because if $B \in f^{-1}(L)$ it would also be that $S \in f^{-1}(L)$, for $f^{-1}(L)$ is a complex. Now, because $M$ is a PL manifold there is a chain

$$
A=C_{0}, C_{1}, \ldots, C_{k}=B
$$

of $n$-simplexes in $J$ such that, for any $i, C_{i}$ and $C_{i+1}$ have a common $(n+1)$-face in $J$. Further, because $A \in f^{-1}(L)$ and $B \in J-f^{-1}(L)$, there is an integer $r<k$ with the property that $C_{r} \in f^{-1}(L)$ and $C_{r+1} \in J-f^{-1}(L)$, and of course $C_{r} \cap C_{r+1}=D$ is an $(n-1)$-simplex of $J$. Also, because $M$ is a PL manifold, $D$ is a face of only these two $n$-simplexes. Consequently, $D$ is a face of exactly one $n$-simplex $C_{r}$ in $f^{-1}(L)$, i.e., a free face of $f^{-1}(L)$. Notice that $D$ is not in $\partial M$, because if it were, $D$ would not be a face of two $n$-simplexes in $J$.

Therefore, $f(D)$ does not belong to the image of $\partial M$ under $f$, and thus $f(D)$ is a free face of $L$.

Let $A_{1}, A_{2}, \ldots, A_{m}$ be the $n$-simplexes of $L$ such that $A_{1}=f\left(C_{r}\right)$. Then $f(D)=B_{1}$ is a face of $A_{1}$ and $L$ collapses simplicially from $B_{1}$ to $L-\left\{A_{1}, B_{1}\right\}$. But $L-\left\{A_{1}, B_{1}\right\}$ is again a proper subcomplex of $K$ having therefore a free face $B_{2}$, belonging to $A_{2}$, say. Therefore $L-\left\{A_{1}, B_{1}\right\}$ collapses simplicially to $L-\left\{A_{1}, B_{1}, A_{2}, B_{2}\right\}$. Continuing inductively on the number $m$ of $n$-simplexes in $L$ we collapse away all $n$-simplexes of $L$, which completes the proof that $L$ collapses to an $(n-1)$-dimensional subcomplex, and therefore $Y$ to an $(n-1)$-dimensional subpolyhedron of $X$. 
We will use the regular neighborhood theory as it is presented in Zeeman [24, Chapter 3], and also the notion of link-collapsibility and the relative regular neighborhood theorem as in Hudson-Zeeman [8]. Although the theorem was proven to be false in general (see [18]) in our applications we will need it only in codimensions at least three, for which the additional conditions saving the theorem are satisfied trivially [9].

The general position theory will be also used as in Zeeman [24, Chapter 6]. Let us mention that the general position results can be improved significantly if in particular we map a PL manifold into another PL manifold, both of a high connectivity. This is the content of Irwin's theorem [10], which we quote explicitly because of the important role it will play throughout this paper:

2.3. TheOREM (IRWIN). Let $X$ and $M$ be PL manifolds of dimension $p$ and $m$, respectively. Let $d=2 p-m$. Let $f: X \rightarrow M$ be a map such that $f / \partial X$ is a PL embedding of $\partial X$ into $\partial M$. Then, $f$ is homotopic to a proper PL embedding, keeping $\partial X$ fixed, if the following conditions are satisfied:

(1) $m-p \geqq 3$,

(2) $X$ is d-connected, and

(3) $M$ is $(d+1)$-connected.

There are counterexamples [24, Chapter 8] showing that the theorem is the best possible along these lines.

3. Some sufficient conditions for embedding in $E^{2 n}$. From the AlexanderNewman theorem [1] it follows easily that the union of two PL $n$-balls, meeting one another in a common face, is a PL ball. The same is true for the difference of two PL $n$-balls. Namely,

3.1. Proposition. Let $A$ and $B$ be $P L n$-balls, such that $B \subset A$ and $\partial B \cap \partial A$ is $a$ $P L(n-1)$-ball. Then $\mathrm{Cl}(A-B)$ is a PL $n$-ball.

Proof. Immediate, using Alexander-Newman theorem.

3.2. THEOREM. Let $X$ be an n-polyhedron, and $Y$ a subpolyhedron of $X$ such that $X$ collapses to $Y$. Then, every PL embedding of $Y$ into $E^{2 n}$ can be extended to a PL embedding of $X$ into $E^{2 n}$.

Proof. The case $n=1$ is obvious, so we can suppose $n \geqq 2$. The proof is by induction on the number of elementary collapses from $X$ to $Y$.

Suppose first that $X=Y \cup A$, where $A$ is a $k$-ball, $k \leqq n$, and $Y \cap A=B$ is a $(k-1)$-ball contained in $\partial A$. Let $f: Y \rightarrow E^{2 n}$ be any PL embedding of $Y$ in $E^{2 n}$. Then, by general position arguments, $f$ extends to the boundary of $A$, i.e., there is an embedding $g: Y \cup \partial A \rightarrow E^{2 n}$ such that $g / Y=f$. We want to extend $g$ over $X$ in such a way that the new map would be 1-1 on $A$. But because of Gugenheim's theorem [7] there is a homeomorphism $t$ of $E^{2 n}$ such that

$$
\operatorname{tg}: Y \cup \partial A \rightarrow E^{2 n}
$$


embeds the $(n-1)$-skeleton of a triangulation of $Y \cup \partial A$ into the canonical subspace $E^{2 n-1} \subset E^{2 n}$. Now, if $v \in E^{2 n}-E^{2 n-1}$ is any point, we can extend $\operatorname{tg} / \partial A$ conewise to $A \rightarrow v * \operatorname{tg}(\partial A)$ obtaining a map $k: X \rightarrow E^{2 n}$ being 1-1 on $A$ and such that $k / Y \cup \partial A=\operatorname{tg}$. Then

$$
h=t^{-1} k: X \rightarrow E^{2 n}
$$

is a PL map such that $h / Y \cup \partial A=g$ and $h / A$ is $1-1$. Finally, from general position considerations, it follows that $h$ can be chosen such that the singular set $S(h)$ contains at most a finite number of points of $X$. Consider

$$
S(h) \cap A=\left\{x_{1}, x_{2}, \ldots, x_{r}\right\} .
$$

Obviously each $x_{i} \in \operatorname{Int} A$. We would like to "pipe away" these singularities.

Let $\left\{y_{1}, y_{2}, \ldots, y_{r}\right\}$ be a set of points chosen in the "free face" $\partial A-B$ of $\partial A$. For each $i=1,2, \ldots, r$ let us join $x_{i}$ with the corresponding $y_{i}$ by a PL arc $\alpha_{i}$ which lies, except for $y_{i}$, in Int $A$. Because $n \geqq 2$ this can be done such that $\alpha_{i} \cap \alpha_{j}=0$ for $i \neq j$. Pick regular neighborhoods $N_{i}$ of $\alpha_{i}$ in $A$ such that

$$
N_{i} \cap N_{j}=0, \quad N_{i} \cap B=0
$$

for $i \neq j$. Because $\alpha_{i}$ is collapsible, $N_{i}$ is a $k$-ball, having a face in $\partial A-B$. Then, by Proposition 3.1, we have that

$$
C=\mathrm{Cl} A\left(-\bigcup_{i} N_{i}\right)
$$

is a PL ball and $C \cap Y=B$. Define the PL homeomorphism $e: X \rightarrow C \cup Y$ such that $e / Y=$ identity (by double extension: identity $B \rightarrow B$ to the homeomorphism $\partial A \rightarrow \partial C$ and then this one to the homeomorphism $A \rightarrow C)$. Finally, the composition $h e: X \rightarrow E^{2 n}$ is an embedding of $X$ into $E^{2 n}$, and $h e / Y=f$.

Because the inductive step is the same as the first step, this completes the proof.

If $Y \subset X$ is a subpolyhedron of $X$ and $X$ collapses to $Y$, we will say that $Y$ is a spine of $X$.

3.3. COROLlaRY. Every n-polyhedron with a lower dimensional spine PL embeds into $E^{2 n}$.

3.4. REMARK. One could expect the following sort of generalization of Theorem 3.2: If $X^{n} \searrow Y$ and $Y$ embeds into $E^{m}, m \leqq 2 n$, then also $X$ embeds into $E^{m}$. Unfortunately this is not true without additional conditions (see Theorem 5.1), because of the existence of (even) a collapsible $n$-polyhedron, which does not embed into $E^{2 n-1}$. The example is as follows:

Let $Y$ be any $(n-1)$-polyhedron which does not embed into $E^{2 n-2}$. Let $X=v * Y$ be a cone on $Y$. Then $X$ is collapsible. We claim that $X$ does not embed into $E^{2 n-1}$. To the contrary, suppose that such an embedding $f: X \rightarrow E^{2 n-1}$ exists. Then, because $f(X) \searrow 0$, a regular neighborhood $N$ of $f(X) \bmod f(Y)$ in $E^{2 n-1}$ will be a $(2 n-1)$-ball and

$$
f(Y) \subset \partial N=(2 \dot{n}-2) \text {-sphere }
$$

which contradicts the assumption that $Y$ cannot be embedded into $E^{2 n-2}$. 
3.5. Proposition. Let $X \subset E^{m}$ be any polyhedron, and let $N$ be a regular neighborhood of $X$ in $E^{m}$. Then $E^{m}-X$ and $E^{m}-$ Int $N$ are of the same homotopy type.

Proof. Because $N$ is a manifold, $M=E^{m}-$ Int $N$ is also a manifold with boundary $\partial M=\partial N$. Further, it is known [24, Chapter 3] that $N-X$ is PL homeomorphic to the open collar $\partial N \times[0,1)$. Therefore $E^{m}-X$ deformation retracts to $E^{m}-\operatorname{Int} N$, which completes the proof.

In codimension at least three, the proposition is also true for $N$ being a relative regular neighborhood of $X$.

Now, we are in the position to prove the main theorem of this section:

3.6. THEOREM. Let $X$ be an $n$-polyhedron with $n \neq 2$. If some triangulation of $X$ contains an $n$-simplex $A$ such that $H^{n}(X-\operatorname{Int} A)=0$, then $X$ PL embeds into $E^{2 n}$.

Proof. If $n=1$, from $H_{1}(X-\operatorname{Int} A)=H^{1}(X-\operatorname{Int} A)=0$, it follows that $X-A$ is contractible, and therefore embeds into $E^{2}$. Moreover, a contractible polyhedron does not separate $E^{2}$, and therefore $X$ embeds into $E^{2}$. So we can suppose $n \geqq 3$.

Let $K$ be a triangulation of $X$ and $A_{1}, A_{2}, \ldots, A_{k}, n$-simplexes of $K$, enumerated in such a way that $A_{k}=A$ is the distinguished $n$-simplex, for which $H^{n}\left(X-\operatorname{Int} A_{k}\right)$ $=0$. Let $X_{0}$ be the subpolyhedron of $X$ determined by the $(n-1)$-skeleton of $K$. For every $i=1,2, \ldots, k$ we define the polyhedron $X_{i}$ by

$$
X_{i}=X_{0} \cup A_{1} \cup \cdots \cup A_{i} .
$$

We will prove that $X$ embeds into $E^{2 n}$ by induction on $i$. We start with $X_{0}$ which embeds into $E^{2 n}$ (even into $E^{2 n-1}$ ). Supposing inductively that $X_{i-1}$ can be embedded into $E^{2 n}$, we have to show that $X_{i}$ embeds into $E^{2 n}$.

Let $K^{\prime}$ be a subdivision of $K$ such that $B_{i}$ is an $n$-simplex in $K^{\prime}$, lying in the interior of $A_{i}$. Then, $A_{i}-$ Int $B_{i}$ collapses to $\partial A_{i}$, and therefore $Y_{i}$ collapses to $X_{i-1}$, where $Y_{i}$ denotes $X_{i}-$ Int $B_{i}$. Now it follows, from Theorem 3.2 and the inductive assumption, that there exists an embedding $f: Y_{i} \rightarrow E^{2 n}$. It remains to show that $f$ extends over $B_{i}$, i.e., to an embedding $X_{i} \rightarrow E^{2 n}$, which completes the proof by induction on $i$.

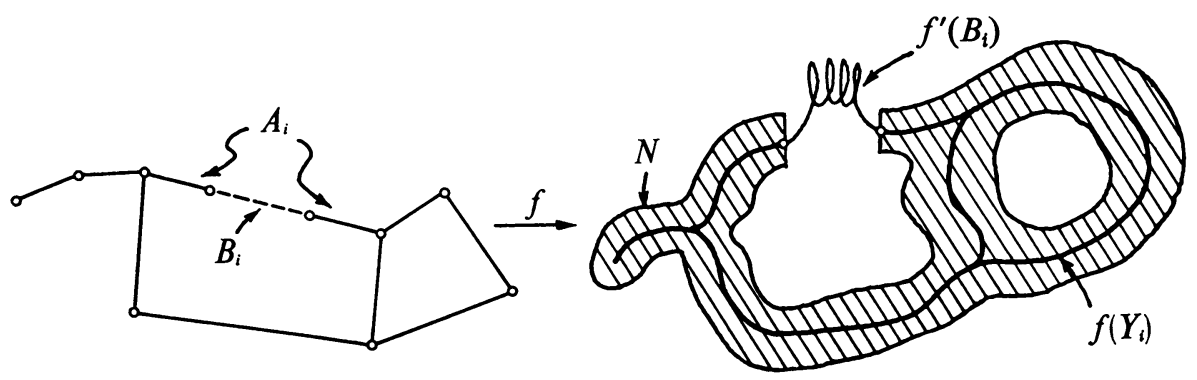

FIGURE 1 
Let $N$ be a regular neighborhood of $f\left(Y_{i}\right) \bmod f\left(\partial B_{i}\right)$ in $E^{2 n}$. By definition this means that $N$ is a PL $2 n$-manifold with boundary, which collapses to $f\left(Y_{i}\right)$, and such that

$$
N \cap f\left(\partial B_{i}\right)=\partial N \cap f\left(\partial B_{\imath}\right)=f\left(\partial B_{i}\right)
$$

The existence of $N$ follows from the fact that $f\left(Y_{i}\right)$ is link-collapsible [8] on $f\left(\partial B_{i}\right)$, because $\partial B_{i} \subset \mathrm{Bd}\left(A_{i}-\operatorname{Int} B_{i}\right)$ and $A_{i}-$ Int $B_{i}$ is a manifold (Figure 1).

Let $M=E^{2 n}-$ Int $N$. Then $M$ is a PL $2 n$-manifold with the boundary $\partial M=\partial N$. Suppose that $M$ is $(n-1)$-connected. Then the embedding $f / \partial B_{i}: \partial B_{i} \rightarrow \partial M$ can be extended to the map $f^{\prime}: B_{i} \rightarrow M$. Further, because $n \geqq 3, f^{\prime} / \partial B_{i}=f$ and $B_{i}$ is contractible, we can apply Irwin's theorem (Theorem 2.3) and replace $f^{\prime}$ by a proper embedding $g: B_{i} \rightarrow M$ such that $g / \partial B_{i}=f^{\prime} / \partial B_{i}$. Finally, $h: X_{i} \rightarrow E^{2 n}$ defined by

$$
\begin{aligned}
h(x) & =f(x) & & \text { for } x \in Y_{i}, \\
& =g(x) & & \text { for } x \in B_{i}
\end{aligned}
$$

embeds $X_{i}$ into $E^{2 n}$, and we are finished.

So, it remains to justify the assumption that $M$ is $(n-1)$-connected. By Proposition 3.5 this is the same as to say that $U=E^{2 n}-f\left(Y_{i}\right)$ is $(n-1)$-connected. First of all, if $S \subset U$ is any (singular) $(n-2)$-sphere, by the $(n-2)$-connectivity of $E^{2 n}$, there is a singular disk $D^{n-1}$ such that $\partial D=S$. But because of general position, $D$ can be chosen to avoid $f\left(Y_{i}\right)$, and consequently $U$ is at least $(n-2)$-connected.

Further, from $X=X_{k}=X_{k-1} \cup A_{k}$ follows $X_{k-1}=X-\operatorname{Int} A_{k}$, and then, by assumption,

$$
H^{n}\left(X_{k-1}\right)=H^{n}\left(X-\operatorname{Int} A_{k}\right)=0 .
$$

Now, because $\operatorname{dim} X=n$ we have $H^{n+1}\left(X_{k-1}, X_{i-1}\right)=0$, and from the following portion

$$
\cdots \rightarrow H^{n}\left(X_{k-1}\right) \rightarrow H^{n}\left(X_{i-1}\right) \rightarrow H^{n+1}\left(X_{k-1}, X_{i-1}\right) \rightarrow \cdots
$$

of the exact cohomology sequence for the pair $\left(X_{k-1}, X_{i-1}\right)$ we conclude that $H^{n}\left(X_{i-1}\right)=0$. Then, by Alexander's duality theorem [17] we have

$$
H_{n-1}(U)=H_{n-1}\left(E^{2 n}-f\left(Y_{i}\right)\right)=H^{n}\left(f\left(Y_{i}\right)\right)=H^{n}\left(Y_{i}\right)=H^{n}\left(X_{i-1}\right)=0
$$

because $Y_{i}$ deformation retracts to $X_{i-1}$. Finally, because $U$ is already $(n-2)$ connected and $n \geqq 3$, we have by the absolute Hurewicz isomorphism theorem [17],

$$
\Pi_{n-1}(U)=H_{n-1}(U)=0
$$

and therefore $U$ is $(n-1)$-connected.

It is not known if the theorem is true or not for $n=2$, even in the case when $X$ satisfies stronger hypothesis. Namely, it is a famous open problem as to whether every contractible 2-polyhedron embeds into $E^{4}$ or not (depending on Poincaré conjecture, see [3]). 
3.7. QUESTION. Any known $n$-polyhedron nonembeddable into $E^{2 n}$ "carries" a large number of cycles (and cocycles). So there is the question as to whether the condition that $H^{n}(X-\operatorname{Int} A-\operatorname{Int} B)=0$, for some $n$-simplexes $A, B$ in a triangulation of $X$ is sufficient for embedding of $X$ into $E^{2 n}$. The problem can be formulated more generally: Determine the function $f(n)=k$ from the set of positive integers into itself, where $k$ is defined to be the largest number such that, if $X$ is any $n$ polyhedron with the property

$$
H^{n}\left(X-\bigcup_{i=1}^{k} \operatorname{Int} A_{i}\right)=0
$$

for a certain choice $A_{1}, \ldots, A_{k}$ of $n$-simplexes in a triangulation of $X$, then $X$ PL embeds into $E^{2 n}$.

As an immediate consequence of Theorem 3.6 we have the following well-known theorem [23]:

3.8. THEOREM. Let $X$ be an n-polyhedron with $n \neq 2$, such that $H^{n}(X)=0$, then, $X P L$ embeds into $E^{2 n}$.

Proof. Let $A$ be any $n$-simplex in the triangulation of $X$. Then, from the exactness of the cohomology sequence

$$
\cdots \rightarrow H^{n}(X)-H^{n}(X-\operatorname{Int} A) \rightarrow H^{n+1}(X, X-\operatorname{Int} A) \rightarrow \cdots
$$

for the pair $(X, X-\operatorname{Int} A)$, it follows that $H^{n}(X-\operatorname{Int} A)=0$, and Theorem 3.6 applies.

Also, the embedding theorem for PL manifolds (e.g. [23]) can be easily extended to all categories of manifolds defined in $\$ 2$. We have the following:

3.9. THEOREM. Every n-manifold $X$ with singular boundary (in particular, every n-dimensional pseudomanifold, polyhedral homology manifold and triangulated manifold) $P L$ embeds into $E^{2 n}$.

Proof. The case $n=1$ is trivial, because the only 1-manifolds with singular boundary are either a segment or a circle. We postpone the proof in the case $n=2$ for $\$ 4$, where it will be proven in even stronger form (see Theorem 4.6), and suppose $n \geqq 3$.

Let $K$ be a triangulation of $X$ and $A$ any $n$-simplex in $K$. Then $X-\operatorname{Int} A$ is a proper subpolyhedron of $X$ and by Lemma 2.2 collapses to the $(n-1)$-dimensional subpolyhedron $Y$. Therefore

and Theorem 3.6 applies.

$$
H^{n}(X-\operatorname{Int} A)=H^{n}(Y)=0
$$

For further applications we need the following:

3.10. Lemma. Let $X_{1}$ and $X_{2}$ be subpolyhedra of an n-polyhedron $X$ such that

$$
X_{1} \cup X_{2}=X, \quad \operatorname{dim}\left(X_{1} \cap X_{2}\right) \leqq n-2 .
$$


If each of $X_{1}$ and $X_{2} P L$ embeds into $E^{2 n}$, then $X P L$ embeds into $E^{2 n}$.

Proof. Let $X_{1} \cap X_{2}=Y$. For each $i=1,2$ we have by assumption an embedding

$$
f_{i}: X_{i} \rightarrow E^{2 n} \subset S^{2 n} \text {. }
$$

Let $C_{i}$ be a cone on $f_{i}(Y)$ in $S^{2 n}$. From the fact that $\operatorname{dim} Y \leqq n-2$, it follows that $\operatorname{dim} C_{i} \leqq n-1$. Consequently, $C_{i}$ can be chosen to be in general position with respect to $f_{i}\left(X_{i}\right)$, namely such that

$$
f_{i}\left(X_{i}\right) \cap C_{i}=f_{i}(Y) .
$$

Because every cone is link-collapsible on its base, there is a regular neighborhood $N_{i}$ of $C_{i} \bmod f_{i}\left(X_{i}\right)$ such that

$$
N_{i} \cap f_{i}\left(X_{i}\right)=\partial N_{i} \cap f_{i}\left(X_{i}\right)=f_{i}(Y)
$$

by [8]. Further, because the cone is collapsible, $N_{i}$ is a PL $2 n$-ball. Let

$$
B_{i}=\mathrm{Cl}\left(S^{2 n}-N_{i}\right) \text {. }
$$

Then, by the Alexander-Newman theorem, $B_{i}$ is also a PL $2 n$-ball and obviously

$$
f_{i}\left(X_{i}\right) \subset B_{i}, \quad f_{i}\left(X_{i}\right) \cap \partial B_{i}=f_{i}(Y) .
$$

Thus we have proved that each of $X_{1}$ and $X_{2}$ can be embedded into $2 n$-ball, having only the image of $Y$ in the boundary of the ball.

Let $S$ be an equator of $S^{2 n} ; D_{1}$ and $D_{2}$ the closed northern and southern hemispheres of $S^{2 n}$ respectively. Let, for $i=1,2, g_{i}: B_{i} \rightarrow D_{i}$ be PL homeomorphisms onto $D_{i}$. Then $g_{i} f_{i}\left(X_{i}\right) \cap S=g_{i} f_{i}(Y)$ and we have two embeddings of $Y$ into $S$. Because

$$
2 \cdot \operatorname{dim} Y+2 \leqq 2(n-2)+2=2 n-2<2 n-1=\operatorname{dim} S,
$$

Gugenheim's unknotting theorem [7] applies and we have a homeomorphism $e^{\prime}: S \rightarrow S$ such that $e^{\prime} g_{1} f_{1} / Y=g_{2} f_{2} / Y$. We extend $e^{\prime}$ to the PL homeomorphism $e: D_{1} \rightarrow D_{1}$. Finally, $h: X \rightarrow S^{2 n}$ defined by

$$
\begin{aligned}
h(x) & =e g_{1} f_{1}(x) & & \text { for } x \in X_{1}, \\
& =g_{2} f_{2}(x) & & \text { for } x \in X_{2}
\end{aligned}
$$

is a $\mathrm{PL}$ embedding of $X$ into $S^{2 n}$ (and therefore into $E^{2 n}$ ).

The assumptions in Lemma 3.10 cannot be weakened because if it held for $\operatorname{dim}\left(X_{1} \cap X_{2}\right)=n-1$, we would be able to prove, by induction on the number of $n$-simplexes in a triangulation of $X$, that every $n$-polyhedron $X$ embeds into $E^{2 n}$.

Suppose that for the $n$-polyhedron $X$ there is a triangulation $K$ such that every $(n-1)$-simplex of $K$ is a face of at most two $n$-simplexes in $K$. Then, it is easy to see that the same is true for any other triangulation of $X$. The next theorem was firstly proved by Van Kampen [19], [20] and reproved by Wu [23]. Here is an easy proof, based on Lemma 3.10: 
3.11. TheOrem. Let $X$ be an n-polyhedron such that every $(n-1)$-simplex in a triangulation of $X$ is a face of at most two n-simplexes. Then, $X$ PL embeds into $E^{2 n}$.

Proof. Let $K$ be a triangulation of $X$. Let $Y$ be an $n$-dimensional homogeneous subpolyhedron of $X$, i.e., the polyhedron determined by all $n$-simplexes of $K$, together with their faces. Suppose that $Y$ embeds into $E^{2 n}$. Then, it follows from general position arguments that the whole polyhedron $X$ can be embedded into $E^{2 n}$, because $\mathrm{Cl}(X-Y)$ is a polyhedron of dimension less than $n$.

Therefore, the problem is how to embed $Y$. But because of the assumption of the theorem, there is the unique decomposition

$$
Y=Y_{1} \cup Y_{2} \cup \cdots \cup Y_{m}
$$

of $Y$, such that each $Y_{i}$ is an $n$-dimensional pseudomanifold, and for $i \neq j, Y_{i} \cap Y_{j}$ consists of simplexes in $K$ of dimension at most $(n-2)$ [2, p. 191].

The proof is by induction on the number $m$ of pseudomanifolds in the decomposition above. If $m=1$, the assertion is true by Theorem 3.9. Now, if $m$ is arbitrary, we can suppose inductively that

$$
Z=Y_{1} \cup Y_{2} \cup \cdots \cup Y_{m-1}
$$

embeds into $E^{2 n}$. Then, because $\operatorname{dim}\left(Z \cap Y_{m}\right) \leqq n-2$, Lemma 3.10 applies, and therefore $Y$ can be embedded into $E^{2 n}$, which completes the proof.

We say that an abelian group is indecomposable, if it cannot be written as a direct sum of its nontrivial subgroups. It is easy to see that a finite cyclic group $Z_{m}$ is indecomposable if its order $m$ is a power of a prime number. We have the following:

3.12. THEOREM. Let $X$ be an n-polyhedron with $n \neq 2$ and such that $H^{n}(X)=Z_{p^{k}}$, where $p$ is prime and $k$ any positive integer. Then, $X P L$ embeds into $E^{2 n}$.

Proof. We can suppose $n \geqq 3$, because no 1 -polyhedron with the required property exists.

Let $K$ be a triangulation of $X$. By Theorem 3.6 it suffices to prove that $K$ contains a simplex $A$ such that $H^{n}(X-\operatorname{Int} A)=0$. Let $A_{1}, \ldots, A_{m}$ be $n$-simplexes in $K$ and let

$$
K=K^{n-1} \cup A_{1} \cup \cdots \cup A_{m}
$$

where $K^{n-1}$ is the $(n-1)$-skeleton of $K$. The proof is by induction on the number $m$ of $n$-simplexes in $K$. If $m=1$ the statement is true, because in this case $X$-Int $A$ is an $(n-1)$-polyhedron.

Let $A$ be any $n$-simplex in $K$. If $H^{n}(X-\operatorname{Int} A)=0$, we are finished. So, suppose that $H^{n}(X-\operatorname{Int} A) \neq 0$. Consider the subcomplex $L=K-\{A\}$ of $K$, triangulating $X$-Int $A$. Then $L$ contains exactly $m-1 n$-simplexes, and from the cohomology exact sequence for the simplicial pair $(K, L)$

$$
\cdots \rightarrow H^{n}(K) \stackrel{\text { onto }}{\longrightarrow} H^{n}(L) \rightarrow H^{n+1}(K, L) \rightarrow \cdots,
$$


it follows that $H^{n}(L)=Z_{p^{k}} / Z_{p^{l}}=Z_{p^{k+l}}$. So, by the inductive assumption, there is an $n$-simplex $B \in L$ such that

$$
H^{n}(L-B)=H^{n}(X-\operatorname{Int} A-\operatorname{Int} B)=0 .
$$

We want to show that $H^{n}(X-\operatorname{Int} B)=H^{n}(K-B)=0$. For this purpose let us consider the following portion of the exact Mayer-Vietoris cohomology sequence $[17$, p. 239] of the simplicial triad $(K, K-A, K-B)$

$$
\begin{aligned}
\cdots \rightarrow H^{n}(K) \stackrel{\text { onto }}{\longrightarrow} H^{n}(K-A) \oplus H^{n}(K-B) \rightarrow H^{n}((K-A) \cap & (K-B)) \\
& =H^{n}(L-B)=0 .
\end{aligned}
$$

Now, from $H^{n}(K-A) \neq 0$, it follows that $H^{n}(K-B)=0$, because the group $H^{n}(K)$ $=Z_{p^{k}}$ is indecomposable and therefore cannot be mapped homomorphically onto the direct sum of two nontrivial groups.

In view of Theorem 3.12 and perhaps Lemma 3.10 it seems reasonable to make the following

3.13. ConjeCture. If $X$ is an $n$-polyhedron such that $H^{n}(X)$ is without free part, i.e., a direct sum of finite cyclic groups (or weaker: an arbitrary, but single finite cyclic group), then $X$ PL embeds into $E^{2 n}$.

The conjecture seems to be the best possible in this direction, because there exist polyhedra possessing a free part in $n$-cohomology which embed and also ones which do not embed into $E^{2 n}$. For example, it is an open question as to whether the condition $H^{n}(X)=Z$ is sufficient for embedding $X$ into $E^{2 n}$.

3.14. REMARK. All the results in this section are obviously true if we replace the ambient space $E^{2 n}$ by any PL $2 n$-manifold, with or without boundary. Namely each of embeddings we considered can be realized as an embedding into an open ball in the structure of given manifold. But the appropriate (and harder) question for this general situation is under which conditions is any map of a polyhedron into the manifold homotopic or even ambient isotopic to a (proper) embedding.

4. Strong embeddability. We say that a polyhedron $X$ strongly embeds into a given PL manifold $M$ if any PL embedding of any subpolyhedron of $X$ into $M$ can be extended to a PL embedding of $X$ into $M$. We will restrict our attention only to the case when the ambient manifold is a Euclidean space.

We observe that the notion of strong embeddability is essentially stronger than the ordinary one. For example, $S^{n}$ embeds into $E^{n+1}$ but does not embed strongly, because an embedding of the equator of $S^{n}$ can knot in $E^{n+1}$ (codimension two!), and therefore does not extend to an embedding of $S^{n}$ into $E^{n+1}$.

4.1. Proposition. Every $n$-polyhedron strongly embeds into $E^{m}$, if $m \geqq 2 n+1$.

Proof. This follows immediately from general position arguments.

Let $S^{p}$ and $S^{a}$ be disjoint PL spheres in $E^{m}$. We say that they are unlinked in $E^{m}$ if there is a PL $m$-ball $B^{m}$ in $E^{m}$ such that $S^{p} \subset B^{m}$ and $S^{q} \subset E^{m}-B^{m}$. Otherwise, 
we say that they are linked or that the pair $\left(S^{p}, S^{q}\right)$ is a link in $E^{m}$. The definition is obviously symmetric. It follows easily from general position arguments that a pair $\left(S^{p}, S^{q}\right)$ is unlinked in $E^{m}$, whenever $m \geqq p+q+2$. On the other hand, links always exist in the first lower dimension, $m=p+q+1$ [24, Chapter 8]. Now it is easy to prove the following:

4.2. Proposition. An n-polyhedron does not embed strongly into $E^{m}$ if $m \leqq 2 n-1$.

Proof. Let $X$ be any $n$-polyhedron, and $A$ an $n$-simplex in a triangulation of $X$. Suppose firstly that $m$ is an odd integer, $m=2 k+1$. In this case we will choose two $k$-spheres $S_{1}^{k}, S_{2}^{k} \subset$ Int $A$ such that they are unlinked in $A$. This is always possible because, from the restriction $m \leqq 2 n-1$, it follows that $k \leqq n-1$. Let

$$
f: S_{1}^{k}+S_{2}^{k} \rightarrow E^{m}
$$

be a PL embedding of the disjoint union $S_{1}^{k}+S_{2}^{k}$ such that the pair $\left(f\left(S_{1}^{k}\right), f\left(S_{2}^{k}\right)\right)$ is a link in $E^{m}$. Suppose that $X$ strongly embeds into $E^{m}$. Then there is a PL embedding $g: X \rightarrow E^{m}$ such that $g / S_{1}^{k}+S_{2}^{k}=f$. Hence, because the property of being unlinked (linked) is a topological invariant, we would have the situation that the spheres $g\left(S_{1}^{k}\right)$ and $g\left(S_{2}^{k}\right)$ are unlinked in $g(A)$ and therefore in $E^{m}$, which contradicts our assumption.

If $m=2 k$ is an even integer, we will choose two unlinked spheres $S_{1}^{k}, S_{2}^{k-1} \subset \operatorname{Int} A$ of dimensions $k$ and $k-1$ respectively, and continue in the same way.

So the only critical dimension in the problem of strong embeddability is the dimension $m=2 n$. Here is a useful condition for this situation:

4.3. TheOREM. Let $X$ be an $n$-polyhedron with $n \neq 2$ and such that $H^{n}(X-\operatorname{Int} A)$ $=0$ for any $n$-simplex $A$ of a triangulation of $X$. Then, $X$ strongly embeds into $E^{2 n}$.

Proof. First of all, $X$ embeds into $E^{2 n}$ by Theorem 3.6. To show that $X$ embeds strongly, let $Y$ be any subpolyhedron of $X$, and $f: Y \rightarrow E^{2 n}$ a PL embedding of $Y$. Let $(K, L)$ be a triangulation of the pair $(X, \mathrm{Cl}(X-Y))$. Then, by general position, $f$ can be extended to the PL embedding $g: X_{0} \rightarrow E^{2 n}$ where $X_{0}$ denotes the union of $Y$ and the polyhedron determined by $(n-1)$-skeleton of $L$. If $L$ does not contain any $n$-simplex, we are finished. Therefore, suppose that $A_{1}, A_{2}, \ldots, A_{k}$ are $n$ simplexes in $L$, and define for each $i=0,1, \ldots, k$ the polyhedron $X_{i}$ as

$$
X_{i}=X_{0} \cup A_{1} \cup \cdots \cup A_{i} .
$$

The proof is by induction on the number $k$. Supposing that there is an embedding $g_{i-1}: X_{i-1} \rightarrow E^{2 n}$ with $g_{i-1} / Y=f$, we will show that $g_{i-1}$ can be extended to $X_{i}$, i.e., over the interior of $A_{i}$.

Let $K^{\prime}$ be a subdivision of $K$ such that $B_{i} \in K^{\prime}$ is an $n$-simplex of $K^{\prime}$ lying in the interior of $A_{i}$. If

$$
Y_{i}=X_{i}-\operatorname{Int} B_{i},
$$


then $Y_{i}$ obviously collapses to $X_{i-1}$, and therefore, by Theorem 3.2, $g_{i-1}$ extends to the embedding $h_{i}: Y_{i} \rightarrow E^{2 n}$. Now, because $Y_{i} \subset X-\operatorname{Int} B_{i}$ and $H^{n}\left(X-\operatorname{Int} B_{i}\right)$ $=0$ by assumption, it follows immediately from the exact cohomology sequence for the pair $\left(X-\operatorname{Int} B_{i}, Y_{i}\right)$ that $H^{n}\left(Y_{i}\right)=0$. Therefore, because also $n \neq 2$ by assumption, we are in a position to apply the construction described in the proof of Theorem 3.6 to obtain an embedding $g_{i}: X_{i} \rightarrow E^{2 n}$ such that $g_{i} / Y_{i}=h_{i}$.

We finish the induction on $k$ by constructing the embedding

$$
g_{k}: X_{k}=X \rightarrow E^{2 n}
$$

with $g_{k} / Y=f$, which completes the proof.

4.4. QUESTION. Is the condition that $H^{n}(X-\operatorname{Int} A)=0$ for every $n$-simplex of a triangulation of $X$ even necessary for strong embeddability of $X$ into $E^{2 n}$ ?

4.5. Corollary. Let $X$ be any $n$-polyhedron with $n \neq 2$ such that $H^{n}(X)=0$ (in particular, any contractible, or more generally, acyclic polyhedron). Then, $X$ strongly embeds into $E^{2 n}$.

Next, we can strengthen Theorem 3.9:

4.6. THEOREM. Every n-manifold $X$ with singular boundary (in particular, every n-dimensional pseudomanifold, polyhedral homology manifold, triangulated manifold and PL manifold) strongly embeds into $E^{2 n}$.

Proof. Suppose first that $n \neq 2$. If $A$ is any $n$-simplex in a triangulation of $X$, by Lemma 2.2, $X$-Int $A$ collapses to an (n-1)-dimensional subpolyhedron of $X$ and therefore $H^{n}(X-\operatorname{Int} A)=0$. So Theorem 4.3 applies.

It is a little harder to prove the theorem in the case $n=2$. Let $Y$ be any proper subpolyhedron of the 2-polyhedron $X$, and $f: Y \rightarrow E^{4}$ a PL embedding. Similarly as in the proof of Theorem 4.3, we consider a pair $(K, L)$, triangulating $(X, \mathrm{Cl}(X-Y))$, and extend $f$ to $g: X_{0} \rightarrow E^{4}$.

Further, if $A_{1}, A_{2}, \ldots, A_{k}$ are 2-simplexes of $L$, we will define

$$
X_{i}=X_{0} \cup A_{1} \cup \cdots \cup A_{i}
$$

and suppose inductively that there is an embedding $g_{i-1}: X_{i-1} \rightarrow E^{4}$ such that $g_{i-1} / Y=f$. Then, if $B_{i} \subset$ Int $A_{i}$ is any 2-simplex, $Y_{i}=X_{i}-\operatorname{Int} B_{i}$ collapses to $X_{i-1}$ and therefore, by Theorem $3.2 g_{i-1}$ extends to $h_{i}: Y_{i} \rightarrow E^{4}$. To complete the proof, we have to extend $h_{i}$ over $B_{i}$.

Let $N$ be a regular neighborhood of $h_{i}\left(Y_{i}\right) \bmod h_{i}\left(\partial B_{i}\right)$ in $E^{4}$. Because $Y_{i}$ is a proper subpolyhedron of $X$, by Lemma 2.2, $Y_{i}$ and then $h_{i}\left(Y_{i}\right)$ collapses to a 1-dimensional subpolyhedron of $h_{i}\left(Y_{i}\right)$, and therefore $N$ is a 4-ball with 1-handles attached on it. Furthermore, because 1-polyhedra unknot in $E^{4}$, from the uniqueness part of the regular neighborhood theorem (codimension three!), it follows immediately that $N$ unknots setwise, i.e., that it can be embedded in essentially 
only one way in $E^{4}$. Considering $E^{4} \subset S^{4}$, from this fact it follows that $M=S^{4}-$ Int $N$ is a 4-ball with 2-handles attached on its boundary. Obviously

$$
S=h_{i}\left(\partial B_{i}\right) \subset \partial M .
$$

It remains to see that $S$ bounds a real 2-disk in $M$. Suppose that $S$ lies in the boundary of a 4-ball contained in $M$. Then $h_{\mathfrak{i}} / \partial B_{\mathfrak{i}}$ can be extended to the embedding $\bar{h}_{i}: B_{i} \rightarrow M$ of a 2 -disc $B_{i}$, by a cone extension, picking a vertex for a cone in the interior of a 4-ball. Finally, $g_{i}: X_{i} \rightarrow E^{4}$ defined by

$$
\begin{aligned}
g_{i}(x) & =h_{i}(x) & & \text { for } x \in Y_{i}, \\
& =h_{i}(x) & & \text { for } x \in B_{i}
\end{aligned}
$$

is a PL embedding of $X_{i}$ into $E^{4}$ with $g_{i} / Y=f$, which completes the inductive step.

So, it remains to justify the assumption that $S$ lies in the boundary of a 4-ball contained in $M$. As we said, $M$ is a 4-ball with 2-handles, i.e., a PL manifold with boundary such that

$$
M=B^{4} \cup \sum_{1}^{r} H_{\imath}
$$

where each $H_{i} \approx I^{4}=I^{2} \times I^{2}, H_{i} \cap H_{j}=0$ for $i \neq j$ and $H_{i} \cap B^{4}=H_{i} \cap \partial B^{4} \approx S^{1} \times I^{2}$. Consider the boundary $I^{2} \times S^{1}$ of the "belt part" of $H_{i}$. Let, for each $i, a_{i} \in \operatorname{Int} I^{2}$, and further let

$$
C_{i}=a_{i} \times S^{1} \subset I^{2} \times S^{1}
$$

be the corresponding "fiber".

Notice, that $S$ can be embedded very badly in $\partial M$. But because of general position we can always assume that

$$
S \cap\left(\bigcup_{i} C_{i}\right)=0
$$

If this is not the case, we will ambient isotope $S$ in $\partial M=\partial N$ into general position with respect to $\cup C_{i}$, and extend this isotopy to an isotopy of a collar $C=\partial N \times I$, keeping $\partial N \times\{1\}$ fixed, and taking care that $C$ is small enough, such that $f(Y)$ remains in $N$-Int $C$.

Now, let $a_{i} \in D_{i} \subset$ Int $I^{2}$, where $D_{i}$ is a PL 2-ball, small enough that it is still the case that $\left(D_{i} \times S^{1}\right) \cap S=0$. Let us notice that $D_{i} \times S^{1} \subset I^{2} \times S^{1}$ is a subset of $D_{i} \times I^{2} \subset I^{2} \times I^{2}=H_{i}$. Consider

$$
\begin{aligned}
H_{i}-\left(\text { Int } D_{i} \times I^{2}\right) & =\left(I^{2} \times I^{2}\right)-\left(\operatorname{Int} D_{i} \times I^{2}\right)=\left(I^{2}-\text { Int } D_{i}\right) \times I^{2} \\
& =\left(\partial I^{2} \times I\right) \times I^{2}=\left(S^{1} \times I^{2}\right) \times I=A_{i} \times I
\end{aligned}
$$

where $A_{i}=S^{1} \times I^{2}$ is an "attaching part" of $H_{i}, i=1,2, \ldots, r$. Now,

$$
M^{*}=B^{4} \cup\left(\bigcup_{i} A_{i} \times I\right)
$$


is PL homeomorphic to $B^{4}$ because each of the cylinders $A_{i} \times I$ can be "pushed" into the collar $\partial B^{4} \times I$ [24, Chapter 3]. By our construction it is obvious that $S \subset \partial M^{*}$ and therefore $M^{*} \subset M$ is the PL 4-ball we were looking for.

This completes the proof of Theorem 4.6.

4.7. REMARK. Obviously Theorem 4.6 is also true for any subpolyhedron of a manifold with singular boundary.

5. Embeddings of manifolds into $E^{2 n-1}$. As we have demonstrated in Remark 3.4, the statement generalizing Theorem 3.2 in the natural way is not true without additional conditions. The following theorem gives a sufficient condition under which such a generalization holds:

5.1. THEOREM. Let $X$ be an n-polyhedron, and $Y \subset X$ a subpolyhedron of $X$ such that $X$ collapses to $Y$ and $Y$ embeds into $E^{k}, k \geqq n$. If the link of every vertex of $a$ triangulation of $X$ strongly embeds into $S^{k-1}$, then $X P L$ embeds into $E^{k}$.

Moreover, if $k-n \geqq 3$, any $P L$ embedding of $Y$ into $E^{k}$ can be extended to a PL embedding of $X$ into $E^{k}$.

Proof. Because $X$ collapses to $Y$, there is a triangulation $(K, L)$ of the pair $(X, Y)$, such that $K$ simplicially collapses to $L$. The proof is by induction on the number of elementary collapses from $K$ to $L$.

First, let us assume that

$$
X=Y \cup A, \quad Y \cap A=a * \partial B
$$

where $A$ is an $r$-simplex of $K, r \leqq n, a \in A$ is a vertex of $A$, and $B$ is the $(r-1)$-face of $A$ opposite the vertex $a$. By assumption there is a PL embedding $f: Y \rightarrow E^{k}$ of $Y$ into $E^{k}$. Consider the stars

$$
\text { st }(a, K)=a * l k(a, K) \text { and } \operatorname{st}(a, L)=a * \operatorname{lk}(a, L)
$$

of the vertex $a$ with respect to $K$ and $L$ respectively. Let

$$
C=f[\mathrm{st}(a, L)], \quad D=f[l k(a, L)] .
$$

Because st $(a, L)$ is a cone over $l k(a, L), C$ is (piecewise linearly) a cone on the base $D$, and therefore $C$ is link-collapsible on $D$ (see [8]). Further, because

$$
\mathrm{Cl}(f(Y)-C) \cap C \subset D,
$$

there exists a regular neighborhood $N$ of $C \bmod [D \cup \mathrm{Cl}(f(Y)-C)]$ in $E^{k}$. Being a regular neighborhood of a cone, $N$ is of course a PL $k$-ball, and further

$$
D \subset \partial N, \quad C-D \subset \operatorname{Int} N .
$$

Now, let $e: N \rightarrow \Delta$ be a PL homeomorphism carrying $N$ onto a $k$-simplex $\Delta$. Then, the embedding

$$
e f / l k(a, L): l k(a, L) \rightarrow \partial \Delta=S^{k-1}
$$


can be extended to the embedding $g^{\prime}: l k(a, K) \rightarrow \partial \Delta$ because $l k(a, L) \subset l k(a, K)$ and $l k(a, K)$ strongly embeds into $S^{k-1}$ by assumption. Let $b$ be the barycenter of $\Delta$ and set $g^{\prime}(a)=b$. Then, $g^{\prime}$ extends conewise to the proper embedding

$$
g: \text { st }(a, K) \rightarrow \Delta \text {. }
$$

Finally, $e^{-1} g:$ st $(a, K) \rightarrow N$ embeds properly st $(a, K)$ into $N$ and $e^{-1} g / l k(a, L)=f$. Therefore $h: X \rightarrow E^{k}$ defined by

$$
\begin{aligned}
h(x) & =e^{-1} g(x) & & \text { if } x \in \mathrm{st}(a, K), \\
& =f(x) & & \text { if } x \in \mathrm{Cl}(X-\operatorname{st}(a, K))
\end{aligned}
$$

embeds $X$ into $E^{k}$.

Because the inductive step is the same as the first one, this completes the proof of the first part of Theorem 5.1.

To prove the second part of the theorem, let us notice that we changed the given map $f$ on st $(a, L) \subset Y$. But, if the codimension is at least three, we can keep $f$ unchanged, i.e., extend it to an embedding of $X$. Namely, in this case by Lickorich's unknotting theorem for cones [12], there is a PL homeomorphism $t: \Delta \rightarrow \Delta$ keeping $\partial \Delta$ fixed, and such that $\operatorname{tg} / \mathrm{st}(a, L)=e f / \mathrm{st}(a, L)$. Then, if we redefine $h$ to be

$$
\begin{aligned}
h(x) & =e^{-1} \operatorname{tg}(x) & & \text { for } x \in \mathrm{st}(a, K), \\
& =f(x) & & \text { otherwise, }
\end{aligned}
$$

we have $h / \mathrm{st}(a, L)=e^{-1} \operatorname{tg} / \mathrm{st}(a, L)=e^{-1} e f / \mathrm{st}(a, L)=f / \mathrm{st}(a, L)$, and therefore $h / Y=f$, which completes the proof.

As an immediate consequence of Theorem 5.1 we have the following:

5.2. THEOREM. Every polyhedral homology n-manifold $X$ with nonempty boundary (in particular, every PL n-manifold with nonempty boundary) PL embeds into $E^{2 n-1}$.

Proof. Let $K$ be a triangulation of $X$. Then, $K$ is a pseudomanifold with nonempty boundary ( $(2)$, and therefore there is an $(n-1)$-simplex $B$ in $K$ which is a face of exactly one $n$-simplex $A$ in $K$. Thus, $K$ collapses simplicially to $K-\{A, B\}$, i.e., $X$ collapses to $X-($ Int $A \cup \operatorname{Int} B)$. Furthermore, because $X-($ Int $A \cup \operatorname{Int} B)$ is a proper subpolyhedron of $X$, it collapses by Lemma 2.2 to an $(n-1)$-dimensional subpolyhedron $Y$ of $X$. So $X$ collapses to $Y$ and $Y$ embeds into $E^{2 n-1}$. Finally, the link of every vertex of polyhedral homology $n$-manifold is polyhedral homology $(n-1)$-manifold ( $\$ 2)$, which strongly embeds into $S^{2 n-2}$ by Theorem 4.6. So, Theorem 5.1 applies.

5.3. REMARK. Without additional conditions or restrictions to the dimension the result is the best possible, for a Möbius strip, for example, does not embed into $E^{2}$. Also, the validity of Theorem 5.2 does not extend to manifolds with singular boundary or pseudomanifolds, because in general we cannot expect that the link of a vertex in such a manifold will strongly embed into $S^{2 n-2}$. 
5.4. THEOREM. Let $X$ be a closed polyhedral homology n-manifold (in particular, a closed PL n-manifold), such that $H_{1}(X)=0$. Then $X P L$ embeds into $E^{2 n-1}$.

Proof. First of all, the assumption $H_{1}(X)=0$ implies that $X$ is orientable. Namely, if $X$ were nonorientable, $\Pi_{1}(X)$ would contain a subgroup of index 2 , which contains all the commutators and therefore the commutators subgroup $C=\left[\Pi_{1}(X), \Pi_{1}(X)\right]$. So, we have $H_{1}(X)=\Pi_{1}(X) / C \neq 0$ which contradicts to the assumption.

If $n=1$, the theorem is satisfied vacuously, because the only closed homology 1 -manifold is a circle for which $H_{1}(X)=Z \neq 0$. If $n=2$, we notice that the link of any vertex of $X$ is a closed homology 1-manifold, i.e., a circle, and therefore $X$ is actually a PL 2-manifold. Besides, $X$ is orientable, which implies that $X$ embeds in $E^{3}$ [13, p. 10]. Finally, if $n=3$, the link of a vertex in $X$ is a homology 2-manifold which is a homology 2-sphere, i.e., a real PL 2-sphere, which shows that $X$ is a PL 3-manifold, and therefore embeds into $E^{5}$ by Wall's theorem [21]. So, we can suppose that $n \geqq 4$.

Let $K$ be a triangulation of $X, A$ an $n$-simplex of $K$ and $B=$ st $\left(a, K^{\prime \prime}\right)$, where $a$ is the barycenter of $A$. Let $Y=X-\operatorname{Int} B$. Then, by Proposition 2.1, $Y$ is a polyhedral homology $n$-manifold with boundary $\partial Y=\partial B$, and therefore there is a PL embedding $f: Y \rightarrow E^{2 n-1}$ by Theorem 5.2. It remains to extend $f$ over $B$.

Let $N$ be a regular neighborhood of $f(Y) \bmod f(\partial Y)$ in $E^{2 n-1}$. Such a neighborhood always exists because $A-\operatorname{Int} B$ is a PL manifold with the boundary component $\partial B$ and a manifold is link collapsible on any subpolyhedron of its boundary. Define $M=E^{2 n-1}-$ Int $N$. Then, $M$ is a PL $(2 n-1)$-manifold with boundary $\partial M=\partial N$, and $f(\partial B) \subset \partial M$.

Suppose that $M$ is $(n-1)$-connected. Then, the embedding $f / \partial B: \partial B \rightarrow \partial M \subset M$ can be extended to the map $\bar{f}: B \rightarrow M$ of the $n$-ball $B$ into $M$. Because $n \geqq 4$ we can apply Irwin's theorem (Theorem 2.3) and replace $\bar{f}$ by a proper PL embedding $g: B \rightarrow M$ for which $g / \partial B=f$. Then $h: X \rightarrow E^{2 n-1}$ defined by

$$
\begin{aligned}
h(x) & =f(x) & & \text { for } x \in Y, \\
& =g(x) & & \text { for } x \in B
\end{aligned}
$$

is a PL embedding of $X$ into $E^{2 n-1}$.

So it remains to justify the claim that $M$ is $(n-1)$-connected. By Proposition 3.5 it suffices to show that $U=E^{2 n-1}-f(Y)$ is $(n-1)$-connected. First of all, because of general position arguments, $U$ is $(n-3)$-connected. Further, from the Alexander duality theorem $[17$, p. 296], it follows that

$$
H_{n-2}(U)=H_{n-2}\left(E^{2 n-1}-f(Y)\right)=H^{n}(f(Y))=H^{n}(Y)=0
$$

because $Y$ is a proper subpolyhedron of $X$ and therefore collapses to an $(n-1)$ dimensional subpolyhedron by Lemma 2.2 . Then, because $n \geqq 3$, the Hurewicz isomorphism theorem $\left[17\right.$, p. 398] applies, and we have $\Pi_{n-2}(U)=H_{n-2}(U)=0$, i.e., $U$ is $(n-2)$-connected. 
Finally, applying again the Alexander duality theorem, we have

$$
H_{n-1}(U)=H_{n-1}\left(E^{2 n-1}-f(Y)\right)=H^{n-1}(f(Y))=H^{n-1}(Y) .
$$

Because $H_{1}(X)=0$ by assumption and $n \geqq 3$, from the reduced Mayer-Vietoris sequence for a pair $(Y, A)$ it follows that $H_{1}(Y)=0$. From this, considering the reduced exact homology sequence

$$
\cdots \rightarrow H_{1}(Y) \rightarrow H_{1}(Y, \partial Y) \rightarrow \tilde{H}_{0}\left(\partial Y=S^{n-1}\right) \rightarrow \cdots
$$

for the pair $(Y, \partial Y)$, we have $H_{1}(Y, \partial Y)=0$. Further, since $X$ is orientable by assumption, we can apply the Lefschetz duality theorem for manifolds with boundary (true also for homology manifolds, [17, pp. 278, 298]) obtaining $H^{n-1}(Y)=H_{1}(Y, \partial Y)=0$. Applying again Hurewicz's theorem we have

$$
\Pi_{n-1}(U)=H_{n-1}(U)=0
$$

and we have proven that $U$ and therefore $M$ is really $(n-1)$-connected.

Theorem 5.4. is a slight generalization of Penrose-Whitehead-Zeeman theorem [15] for $k=1$. Namely, we have the following:

5.5. Corollary. Every closed, simply connected PL n-manifold M PL embeds into $E^{2 n-1}$.

The suspension of Poincaré's 3-manifold is an example of an orientable, simply connected homology 4-manifold, which is not a PL manifold, but which embeds into $E^{7}$ by Theorem 5.4 .

Finally, let us mention that Weber [22] proved recently the validity of Theorem 5.4 for orientable PL manifolds without the assumption $H_{1}(X)=0$.

\section{BIBLIOGRAPHY}

1. J. W. Alexander, The combinatorial theory of complexes, Ann. of Math. 31 (1930), 292-320.

2. P. S. Alexandroff and H. Hopf, Topologie. Vol. 1, Springer, Berlin, 1935; reprint, Chelsea, New York, 1965; newer ed., Die Grundlehren der math. Wissenschaften, Band 45, SpringerVerlag, Berlin, 1967. MR 32 \#3023.

3. M. L. Curtis, On 2-complexes in 4-space, Topology of 3-manifolds and related topics (Proc. Univ. of Georgia Inst., 1961), Prentice-Hall, Englewood Cliffs, N. J., 1962, pp. 204-207. MR 25 \#3537.

4. C. H. Edwards, Jr., Unknotting polyhedral homology manifolds, Michigan Math. J. 15 (1968), 81-95. MR 37 \#2218.

5. A. I. Flores, Über die Existenz n-dimensionaler Komplexe, die nicht in den $R^{2 n}$ topologisch einbettbar sind, Erg. Math. Kolloq. 5 (1933), 17-24.

6. - U Uber n-dimensionaler Komplexe, die im $R^{2 n+1}$ absolut selbstverschlungen sind, Erg. Math. Kolloq. 6 (1934), 4-7.

7. V. K. A. M. Gugenheim, Piecewise linear isotopy and embedding of elements and spheres. I, Proc. London Math. Soc. (3) 3 (1953), 29-53. MR 15, 336.

8. J. F. P. Hudson and E. C. Zeeman, On regular neighborhoods, Proc. London Math. Soc. (3) 14 (1964), 719-745. MR 29 \#4063. 
9. L. S. Husch, Jr., Piecewise linear embeddings in codimensions 0 and 1, Doctoral Thesis, Florida State University, Tallahassee, Florida, 1967.

10. M. C. Irwin, Embeddings of polyhedral manifolds, Ann. of Math. (2) 82 (1965), 1-14. MR 32 \#460.

11. R. C. Kirby and L. C. Siebenmann, On the triangulation of manifolds and the Hauptvermutung, Bull. Amer. Math. Soc. 75 (1969), 742-749.

12. W. B. R. Lickorish, The piecewise linear unknotting of cones, Topology 4 (1965), 67-91. MR 34 \#3585.

13. W. S. Massey, Algebraic topology: An introduction, Harcourt, Brace \& World, New York, 1967. MR 35 \#2271.

14. K. Menger, Dimensions theorie, Teubner, Leipzig, 1928.

15. R. Penrose, J. H. C. Whitehead and E. C. Zeeman, Embedding of manifolds in euclidean space, Ann. of Math. (2) 73 (1961), 613-623. MR 23 \#A2218.

16. H. Seifert and W. Threlfall, Lehrbuch der Topologie, Teubner, Leipzig, 1934; reprint, Chelsea, New York, 1947.

17. E. H. Spanier, Algebraic topology, McGraw-Hill, New York, 1966. MR 35 \#1007.

18. R. Tindell, A counterexample on relative regular neighborhoods, Bull. Amer. Math. Soc. 72 (1966), 892-893. MR 33 \#6635.

19. E. R. Van Kampen, Komplexe in euklidische Raumen, Abh. Math. Sem. Univ. Hamburg 9 (1932), $72-78$.

20. - - Berichtung dazu, Abh. Math. Sem. Univ. Hamburg 9 (1932), 152-153.

21. C. T. C. Wall, All 3-manifolds imbed in 5-space, Bull. Amer. Math. Soc. 71 (1965), 566567. MR 30 \#5324.

22. C. Weber, Plongements de polyèdres dans le domaine métastable, Comment. Math. Helv. 42 (1967), 1-27. MR 38 \#6606.

23. Wu Wen-tsün, a) On the realization of complexes in euclidean spaces. I, Sci. Sinica 7 (1958), 251-297. MR 20 \#5471.

b) II, Sci. Sinica 7 (1958), 365-387. MR 22 \#3000.

c) III, Sci. Sinica 8 (1959), 133-150. MR 20 \#4825b.

24. E. C. Zeeman, Seminar on combinatorial topology, Mimeographed Notes, Inst. Hautes Études Sci., Paris, 1963.

UNIVERSITY OF GEORGIA, Athens, Georgia 30601

Institute of Mathematics, University of ZaGreb, Zagreb, Yugoslavia 